\title{
Atendimento à população de rua em um Centro de Saúde Escola na cidade de São Paulo
}

\author{
HEALTH ASSISTANCE TO HOMELESS PEOPLE IN A HEALTH UNIT IN SÃO PAULO CITY \\ ATENCIÓN A LA POBLACIÓN QUE VIVE EN LAS CALLES, EN UN CENTRO DE \\ SALUD DOCENT EN LA CIUDAD DE SÃO PAULO
}

\begin{abstract}
Rhavana Pilz Canônico', Ana Cristina D’Andretta Tanaka², Márcia Maria Porto Roccetto Mazza Maria de Fátima e Souza ${ }^{4}$, Maria Cristina Bernat ${ }^{5}$, Luciana Xavier Junqueira ${ }^{6}$
\end{abstract}

\section{RESUMO}

OCentro de Saúde Escola Geraldo Honório de Paula Souza localizase na Faculdade de Saúde Pública da Universidade de São Paulo, atende à população do território adjacente e contribui para a formação acadêmica na área da saúde. Também oferece assistência aos moradores em situação de rua da região de Pinheiros/Lapa e conta com o trabalho do Programa A Gente na Rua, parceria entre o Centro Social Nossa Senhora do Bom Parto e Secretaria Municipal de Saúde de São Paulo. OPrograma é composto por três agentes comunitários de saúde e uma enfermeira, que realizam atendimento e acompanhamento a essa população no Centro de Saúde com as enfermeiras da unidade. Os agentes abordam os moradores de rua que, posteriormente, serão acompanhados tanto na unidade como nas visitas de rua realizadas pelos próprios agentes e pela enfermeira do Programa, considerando os princípios de equiidade e universalidade do Sistema Único de Saúde (SUS).

\begin{abstract}
Geraldo Honório de Paula Souza health unit is located close to the Public Health College at University de São Paulo and has largely contributed to improve education in the health field. As it registers just population who live in a specific location nearly, it has also provided help to the homeless people in the area of Pinheiros couting on the support of the Program Gente de Rua, a partnership between Social Center Nossa Senhora do Bom Parto and Health Secretary of São Paulo municipality. This program consists of three communitarian agents and a nurse who take care of this population in the health center. The agents first contact homeless people who can be later assisted by a heath team in the unit. Assistance also includes visits by the agents and the nurse from the program, taking into consideration the principle of equity of Brazilian Unified Health System.
\end{abstract}

\section{DESCRITORES}

Sem-teto.

Vulnerabilidade social.

Saúde pública.

Eqüidade.

Atenção primária à saúde.

\section{KEY WORDS}

Homeless persons.

Social vulnerability.

Public health.

Equity.

Primary health care.

\section{RESUMEN}

El centro de Salud Escuela Geraldo Honório de Paula Souza está localizado junto a la Facultad de Salud Pública de la Universidad de São Paulo atendiendo la población del territorio adyacente y contribuyendo para la formación académica en el área de la salud. También ofrece asistencia a los habitantes que viven en las calles de la región de Pinheiros y cuenta con el trabajo del Programa A Gente na Rua, una sociedad entre el Centro Social Nossa Senhora do Bom Parto y la Secretaría Municipal de Salud de São Paulo. Ese programa es compuesto por tres agentes comunitarios de salud $\mathrm{y}$ un enfermero que atienden y observan esa población en el centro de salud junto con las enfermeras de la unidad. Los agentes abordan a los habitantes que viven en las calles que posteriormente serán observados en la unidad y también en las visitas en las calles realizadas por los propios agentes y por lo enfermero del Programa, contemplando el principio de equidad del Sistema Único de Salud.

\section{DESCRIPTORES}

Personas sin hogar.

Vulnerabilidad social.

Salud pública.

Equidad.

Atención primaria de salud.

\author{
1 Enfermeira do \\ Programa de Saúde \\ da Família para \\ População de Rua \\ "Programa A Gente \\ na Rua" - Região \\ Lapa/ Pinheiros. São \\ Paulo, SP, Brasil. \\ mavana_2@yahoo.com.br/ \\ 2 Professora Titular do \\ Departamento de \\ Saúde Materna da \\ Faculdade de Saúde \\ Pública, Universidade \\ de São Paulo Diretora \\ do Centro de Saúde \\ Escola Geraldo de \\ Paula Souza. São \\ Paulo, SP, Brasil. \\ 3 Enfermeira do Centro \\ de Saúde Geraldo de \\ Paula Souza. São \\ Paulo, SP, Brasil. \\ mmprm@usp.br \\ 4 Enfermeira do Centro \\ de Saúde Geraldo de \\ Paula Souza. São \\ Paulo, SP, Brasil. \\ 5 Enfermeira do Centro \\ de Saúde Geraldo de \\ Paula Souza. São \\ Paulo, SP, Brasil. \\ cristina@nemeton.com.br \\ 6 Enfermeira do Centro \\ de Saúde Geraldo de \\ Paula Souza. São \\ Paulo, SP, Brasil.
}




\section{O CENTRO DE SAÚDE}

Em princípios do século XX ainda prevalecia no Brasil um quadro de saúde que se caracterizava pelo predomínio de doenças transmissíveis como cólera, febre amarela, varíola, tuberculose, hanseníase e febre tifóide. Naquela época, havia um grande interesse político e econômico na busca de uma ação sanitarista capaz de inverter o quadro de saúde até então conhecido. Houve inúmeras iniciativas no intuito de sanar o problema, entretanto foram imediatistas, limitadas e impositivas. Entre as iniciativas adotadas na cidade de São Paulo no sentido de reorganização dos serviços sanitários do Estado, foram criadas as Inspetorias de Higiene do Trabalho, de Educação Sanitária, de Profilaxia das Moléstias Infecciosas e os Centros de Saúde. Estes últimos tinham como função de localizar os focos de doenças transmissíveis, a coleta de dados de morbidade e a realização de educação sanitária dos pacientes e suas famílias $^{(1)}$.

O Centro de Saúde Geraldo de Paula Souza (CSEGPS) é o primeiro Centro de Saúde da América Latina a ser criado oficialmente pelo Decreto $n^{\circ} .3874$ de 19/7/1925 nas dependências do antigo Instituto de Higiene, hoje Faculdade de Saúde Pública da Universidade de São Paulo (FSP-USP). Desde então tem exercido suas atividades de ensino, pesquisa e prestação de serviço à comunidade, ininterruptamente. Pertence à FSP-USP e é ligado diretamente à diretoria desta Faculdade ${ }^{(1)}$.

O CSEGPS integra a Coordenadoria Regional de Saúde Centro-Oeste - Supervisão Lapa/ Pinheiros. A população de sua área de abrangência, composta pelos bairros Jardim Paulista e Pinheiros, é de aproximadamente 94.000 habitantes $^{(2)}$.

O Centro de Saúde é referência na cidade São Paulo nas seguintes especialidades: dermatologia sanitária, tuberculose, hanseníase, neuropediatria, geriatria, endocrinologia e no atendimento às vítimas de abuso sexual por meio do Programa de Atenção a Vítimas de Abuso Sexual (PAVAS). Entretanto, atende apenas a população da área de abrangência nos Programas de Saúde do Adulto, Saúde da Mulher, Saúde da Criança, Nutrição, Saúde bucal, Oftalmologia, Saúde Mental, Homeopatia, Acupuntura, Fonoaudiologia, Serviço Social e Atendimento à pessoa em situação de rua. O CSEGPS também possui laboratório de análises clínicas, farmácia, sala de medicação e curativo; grupos educativos realizados por equipe multiprofissional. Como centro de saúde escola, exerce importante papel no ensino e pesquisa, sendo campo de estágio nas áreas de: geriatria, enfermagem, terapia ocupacional, fisioterapia, fonoaudiologia, psicologia, acupuntura, homeopatia.
A Vigilância em Saúde é uma atividade fundamental deste serviço, em que as ações vão desde a notificação compulsória de doenças transmissíveis, sua identificação, captação de casos e comunicantes; supervisão de saúde do idoso incapacitado; até investigação de óbito materno e neonatal. É importante destacar o intenso trabalho realizado com portadores de tuberculose, um serviço bastante compromissado dentro do CSEGPS e que por sua vez, teve a iniciativa de realizar busca ativa com catadores de papelão que trabalhavam em uma Cooperativa próxima da região. Por meio deste trabalho de vigilância, não apenas os catadores, mas os moradores em situação de rua que moravam nas proximidades conheceram o Centro de Saúde Escola. Deuse, então, início ao acolhimento às pessoas em situação de rua.

\section{A POPULAÇÃO EM SITUAÇÃO DE RUA}

A urbanização desordenada, o aumento do desemprego e a extrema desigualdade social são uns dos efeitos negativos da globalização que afetam a cidade de São Paulo. Neste cenário, surge uma população pouco reconhecida, mas muito vista nas ruas, embaixo dos viadutos, nos faróis e becos da cidade. Segundo dados da Fundação e Instituto de Pesquisas Econômicas (FIPE) em 2003, São Paulo tinha aproximadamente 10.500 pessoas em situação de rua, ou seja, vivendo sem qualquer abrigo ou proteção, ao relento, ou em situação de albergue $^{(3)}$. Hoje, acredita-se que haja aproximadamente 13.000 pessoas morando nas ruas, concentrando-se a maior parte na região central da cidade como Sé, República e Santa Cecília. Certamente é uma população que cresce gradativamente.

Ainda é desconhecido o aspecto que mais condiciona a rua como moradia dessas pessoas. Neste contexto tão adverso, a permanência na rua, com toda a violência que nela está imbricada são relatados inúmeros fatores que comprometem sua estrutura individual agravando ainda mais suas capacidades mentais e físicas. Muitas vezes, drogas como o álcool e o crack estão presentes na vida dos moradores de rua, bem como o distúrbio mental. Entretanto, podem ter chegado às ruas por conta desses problemas, ou podem ter adquirido certos hábitos como o consumo abusivo de drogas e o desenvolvimento de muitas doenças, em sua maior parte, mentais, em decorrência de sua permanência neste outro modo de vida. É sabido que algumas pessoas optam por morar na rua. Neste caso a liberdade se sobrepõe às regras da união familiar. O rompimento com a família é extremamente presente na vida dos moradores de rua, sendo prevalentes situações de violência doméstica, sexual e moral. A maioria vive sozinha, mas aglomera-se com outros 
companheiros de praça, ocasionando uma união pelas dificuldades comuns, mas com freqüente desconfiança.

As freqüentes dificuldades vivenciadas por essas pessoas os motivaram a buscar parcerias na rua desencadeando o movimento social, tornando maior a força dessa população para reivindicações.

Depois de anos de luta, os movimentos sociais e populares conseguiram aporte legal na Constituição Federal de 1998 para assistência social. Isso os elevou à condição de política pública, que compõe as políticas sociais da seguridade social. Hoje a população em situação de rua discute suas questões por meio de fóruns compostos por entidades que trabalham com esse grupo populacional e movimentos organizados por eles próprios e ainda realizam suas reivindicações prioritárias tendo como temas os direitos sociais, a segurança, a moradia, o trabalho, a saúde entre outros, no Dia Nacional de Luta do Povo de Rua conhecido pelo Grito dos Excluídos ${ }^{(4)}$.

\section{O PROGRAMA}

\section{A GENTE NA RUA}

Em 23 de julho de 2003, considerado o dia do Grito dos Excluídos, parte das pessoas em situação de rua descontente com seu atendimento nos serviços de saúde foi às ruas da capital para reivindicar maior atenção a sua saúde. Essas pessoas questionavam o preconceito por serem atendidas de forma diferenciada dos demais usuários e que algumas equipes de saúde resistiam em atendê-las em decorrência de sua condição precária de higiene ${ }^{(4)}$.

Baseando-se em dois princípios do Sistema Único de Saúde (SUS), universalidade e equidade, a Secretaria Municipal de Saúde de São Paulo (SMS) juntamente com Centro Social Nossa Senhora do Bom Parto, uma organização social sem fins lucrativos, implantou o Programa A Gente na Rua (PAR) atendendo à reivindicação daquele $\mathrm{ano}^{(4)}$.

O PAR atua em três regiões de maior concentração de população de rua na cidade de São Paulo: Sé, Mooca e Lapa/ Pinheiros. É composto por três equipes de Programa de Agentes Comunitários de Saúde (PACS), com 4 enfermeiras e 35 agentes comunitários de saúde de rua (ACSR); duas assistentes sociais e uma coordenadora ${ }^{(4)}$.

\section{O ATENDIMENTO NO CENTRO DE SAÚDE}

Umas das equipes PACS está inserida no Centro de Saúde Escola Geraldo de Paula Souza com a atuação de uma enfermeira e três ACSR. O trabalho é desenvolvido juntamente com as enfermeiras do centro de saúde.

Os princípios de universalidade e eqüidade do SUS baseiam a inserção do morador em situação de rua no serviço de saúde e a realização de seu atendimento e acompanhamento de acordo com suas necessidades de saúde ${ }^{(5)}$.

Essa inserção do morador em situação de rua no centro de saúde se dá por demanda espontânea, quando ele vai à unidade com queixas tanto físicas como psicológicas; por meio da abordagem do agente que registra as queixas de saúde do indivíduo em fichas de cadastro do PAR e encaminha para o centro de saúde; e, finalmente, por encaminhamento dos profissionais que trabalham nos equipamentos sociais da região.

$\mathrm{O}$ atendimento inicial compreende um acolhimento realizado pelas enfermeiras da unidade, momento em que são levantadas suas necessidades de saúde mais urgentes visando uma melhoria de suas condições de saúde e um encaminhamento adequado para suas queixas, podendo ser uma consulta médica e/ou odontológica, um curativo, uma medicação, entre outros procedimentos realizados no centro de saúde. Freqüentemente os enfermeiros encaminham os moradores para Casa de Convivência e albergue para cuidados com a higiene, alimentação e pernoites.

A parceria com os equipamentos sociais da região é muito importante uma vez que o conceito de saúde hoje é mais ampliado que na época da criação do centro de saúde. É necessário prover não apenas condições de higiene e alimentação, mas tentar resgatar o morador em situação de rua enquanto cidadão, encaminhando-o para outros serviços para que possam retirar sua documentação pessoal. Destaca-se a importância do agente de saúde também neste processo, pois muitas vezes acompanha o morador em serviços como poupa tempo, cartório e banco.

Além das atividades das enfermeiras da unidade, há também a visita de rua realizada pela enfermeira do PACS que vai à rua junto com o agente para abordar o morador e avaliar sua atual condição de saúde e encaminhá-lo de acordo com suas queixas.

É importante ressaltar que o agente de saúde de rua mantém o acompanhamento deste morador após sua passagem pela unidade, dando seqüência à manutenção do vínculo deste indivíduo com o centro de saúde.

O trabalho realizado pelos enfermeiros neste centro de saúde difere do serviço de pronto atendimento na medida em que se valoriza um acompanhamento efetivo desta pessoa. Embora reconhecendo dificuldades para tal prática, podemos enumerar alguns avanços no atendimento a esta parcela da população como: adesão e conclusão do tratamento de tuberculose; adesão ao uso de contraceptivo injetável; controle de pré-natal; participação no grupo de planejamento familiar; sensibilização para o controle de doenças crônicas não-transmissíveis como hipertensão e diabetes contando com o trabalho do agente para o oferecimento e supervisão da medicação; sensibilização para a 
busca do tratamento contra o alcoolismo e drogadição; mobilização dos profissionais de saúde da unidade para o atendimento dessas pessoas.

Esse acompanhamento torna-se efetivo na medida em que se faz a matrícula do morador em situação de rua, obtendo-se um número de prontuário para ele. Mesmo que ele venha a perder seu cartão de matrícula, visto que é uma população susceptível a roubos e perdas, o agente de saúde e a enfermeira do PACS de rua possuem uma relação de nomes com seus respectivos números de prontuários ou ainda, o agente, por muitas vezes, guarda o cartão em uma pasta na unidade. É rotineiro o agente e a enfermeira do PACS localizarem o morador para lembrá-lo de sua consulta. Essas ações fazem com que o vínculo com a equipe e o centro de saúde permaneça, pois o histórico deste morador é preservado e sempre que ele retorna à unidade os profissionais resgatam seu último atendimento e cobram os resultados esperados. Entretanto freqüentemente é necessário lembrar ao morador de rua sobre as condutas tomadas e reforçar que o tratamento e continuação do acompanhamento dependem mais dele que do próprio serviço de saúde.

Mesmo com o trabalho que o centro de saúde realiza, percebe-se que ainda há muita resistência por parte desta população em aderir à unidade de saúde enquanto usuário do SUS e reconhecer que é preciso cuidar da saúde seja para sua subsistência neste modo de vida, seja para sua manutenção enquanto indivíduo bio-psico-social ou até mesmo para ir em busca de seus objetivos, tais como retornar ao mercado de trabalho, à moradia e à família.

\section{CARACTERIZAÇÃO DOS MORADORES DE RUA CADASTRADOS}

Atualmente (ano de 2007), são cadastradas 206 famílias no PAR na região do Centro de Saúde Paula Souza, totalizando 216 pessoas segundo o Sistema de Informação de Atenção Básica (SIAB) da SMS. Esses dados confirmam a grande quantidade de pessoas morando sozinhas nas ruas $^{(6)}$.

Em relação ao sexo, estão cadastradas 129 pessoas do sexo masculino e 87 do sexo feminino, revelando que morar na rua é uma condição vivenciada predominantemente por homens $^{(6)}$.

Das condições referidas no SIAB, 57 pessoas relatam serem alcoolistas, ou seja, 26,0\% dos casos; 14 (6,5\%) pessoas referem hipertensão arterial; cinco pessoas referem diabetes $(2,3 \%)$ e 6 referem tuberculose $(2,8 \%)^{(6)}$.

Especialmente em relação à tuberculose, vale ressaltar que a população em situação de rua é mais propensa a contrair tuberculose pelas condições precárias de higiene e abri- go e, ainda pela facilidade de aglomerarem-se em locais fechados como albergues.

\section{REFLEXÕES E CONSIDERAÇÕES FINAIS}

Os desafios cotidianos enfrentados pelos profissionais de saúde envolvidos neste trabalho motivam-os a inserir esta parcela da população nos serviços, objetivando melhorar suas condições de saúde.

Ainda é necessária maior sensibilização não apenas dos profissionais de saúde, mas de toda a sociedade civil no intuito de compreender a população em situação de rua.

Na perspectiva da enfermagem, o trabalho com a população em situação de rua é um trabalho em construção e desafiador. É preciso aguçar nestes profissionais, não apenas o interesse em atender essa população, mas desenvolver a habilidade da escuta, seu principal instrumento de trabalho. Sem conhecer a história da pessoa que mora na rua, suas adversidades e, sem atentar para suas queixas, certamente não será fácil descobrir suas reais necessidades.

Atualmente, existe certo interesse em pesquisar essas pessoas para conhecer seu modo de vida e identificar suas reais dificuldades. Muitos sociólogos, antropólogos e filósofos estão atuando neste campo, pois se trata de um assunto intrigante - entender porque algumas pessoas vivem de um modo diferente do usual e do que é socialmente esperado. A importância de tais pesquisas pode ajudar no processo de divulgação do problema social e possível elaboração de políticas para essa população.

Não se descarta, no entanto, a problematização que essa população traz para a sociedade civil de um modo geral. A exposição de sua condição de vida pode gerar nas pessoas diferentes reações resultando em sentimentos como piedade, indignação ou até mesmo indiferença.

Certamente também há um interesse político e econômico em que essas pessoas saiam das ruas da cidade, algumas vezes por questões de limpeza urbana, outras pelo fato de incomodarem pedindo esmolas e comidas, ou então pelo perigo que acreditam que possam oferecer à sociedade. Entretanto existem recentes iniciativas do terceiro setor, como as organizações sociais e instituições sem fins lucrativos que visam retirar essas pessoas da rua com objetivo de reinserção social.

Desta forma, não é necessária apenas vontade de se realizar uma política pública eficaz, mas são necessárias ações concretas realizadas pelo poder público, privado e sociedade civil em um conjunto, que considerem todos cidadãos e, principalmente, indivíduos que desejam levar uma vida com qualidade seja ela onde for.

Considerando todo o trabalho realizado neste centro de saúde, a expectativa dos profissionais que atuam diretamente 
com essa população é a construção de um modelo de atenção que contribua para o resgate de sua auto-estima, de sua

\section{REFERÊNCIAS}

1. Bernat MC. Compreensão do processo de trabalho do Enfermeiro de Saúde Pública na Secretaria de Estado da Saúde - Município de São Paulo: convergências e divergências [monografia]. São Paulo: Faculdade Saúde Pública, Universidade de São Paulo; 1999.

2. São Paulo (Cidade). Secretaria Municipal de Saúde. Coordenação de Epidemiologia e Informação (CEINFO-SMS). Supervisão Técnica de Saúde Lapa/ Pinheiros. São Paulo; 2007.

3. Fundação Instituto de Pesquisas Econômicas (FIPE). Censo da População em Situação de Rua. São Paulo; 2003.

4. São Paulo (Cidade). Secretaria Municipal de Saúde. Centro Social Nossa Senhora do Bom Parto. "Programa A Gente na Rua": Relatório de Gestão. São Paulo; 2007. condição enquanto indivíduo produtivo e, finalmente enquanto cidadão brasileiro.

5. Brasil. Lei n. 8080, de 19 de setembro de 1990. Dispõe sobre as condições para Promoção, Proteção e Recuperação da Saúde, a organização e o funcionamento dos serviços correspondentes e dá outras providências [legislação na Internet]. Brasília; 1990 [citado 2007 jul. 15]. Disponível em: http://www.ulbra.br/ processoseletivocanoas/lei8080.pdf

6. São Paulo (Cidade). Secretaria Municipal de Saúde. Centro de Epidemiologia e Informação (CEINFO-SMS). Supervisão Técnica de Saúde Lapa/Pinheiros. Sistema de Informação de Atenção Básica (SIAB). São Paulo; 2007. 